\title{
La competencia científica en las actividades de aprendizaje incluidas en los libros de texto de Ciencias de la Naturaleza
}

\author{
Silvana Perez \\ Facultad de Física. Master Profesional en Enseñanza de Física. Universidad Federal de Pará. Pará. Brasil \\ silperez_1972@hotmail.com \\ ORCID: https:/ / orcid.org/0000-0001-5138-9667 \\ Jesús Ángel Meneses Villagrá \\ Departamento de Didácticas Especificas. Facultad de Educación. Universidad de Burgos. Burgos. España. \\ meneses@ubu.es \\ ORCID: https:// orcid.org/0000-0003-4839-0418
}

[Recibido: 12 Mayo 2019. Revisado: 19 Diciembre 2019. Aceptado: 1 Febrero 2020]

Resumen: Estudios recientes señalan que una amplia mayoría de los profesores de España utilizan el libro de texto en las actividades desarrolladas en el aula. Estos datos apuntan a la importancia de evaluar la calidad de los libros de texto existentes, particularmente en la forma en que incorporan las competencias en el currículo escolar. El presente trabajo analiza las actividades de aprendizaje incluidas en tres editoriales de libros de texto de Ciencias de la Naturaleza de España, para el cuarto curso de la educación primaria, con el objetivo de identificar si la competencia científica está presente en dichos recursos educacionales. Concretamente, se busca identificar las actividades de aprendizaje que exploran metodologías indagatorias, que están directamente vinculadas al trabajo científico y a la comprensión de la naturaleza de la ciencia. Los resultados revelan un patrón similar en las tres editoriales, siendo la dimensión conceptual la más trabajada, y poco explorada la dimensión metodológica e integrada. No fueron encontradas actividades de aprendizaje con abordaje indagatorio, sin embargo, en dos de las tres editoriales se han encontrado algunas propuestas que incluyen diseños de ingeniería, aunque con una orientación no adecuada para un desarrollo correcto por los estudiantes.

Palabras clave: Ciencias Naturales, libros de texto, actividades de aprendizaje, actividades indagatorias

Article title: The learning activities included in the textbooks of Nature Sciences and the development of scientific competences

Abstract: Recent studies indicate that more than $80 \%$ of the Spanish teachers use almost exclusively the
textbook in the activities developed in the classroom. These data point to the importance of evaluating the
quality of existing textbooks, particularly in the way they incorporate basic competencies into the school
curriculum. In this paper, the learning activities included in three Science textbook of Spain are analyzed, for the
fourth year of primary education, with the aim of identifying whether the scientific competence is present. More
specifically, we look for learning activities that explore inquiry methodologies, which are directly linked to
scientific work and to the understanding of the Nature of Science. The results reveal a similar pattern in the three
editorials, the conceptual dimension being the most worked out, and with only a few activities that promote the
development of the methodological and integrate dimensions. No learning activities were found with an inquiry
approach, however in two of the three publishers some proposals have been found that include engineering
designs, although with an inadequate orientation for a correct development by the students. Keywords: Science, textbooks, learning activities, inquiry.

Para citar este artículo: Pérez, S., Meneses Villagrá, J.A. (2020) La competencia científica en las actividades de aprendizaje incluidas en los libros de texto de Ciencias de la Naturaleza Revista Eureka sobre Enseñanza y Divulgación de las Ciencias 17(2), 2101. doi: 10.25267/Rev_Eureka_ensen_divulg_cienc.2020.v17.i2.2101 


\section{Introducción}

El libro de texto, entendido como una herramienta pedagógica de enseñanza-aprendizaje, con una determinada distribución y jerarquización de ideas, intrínsecamente relacionada con una recreación del conocimiento epistémico (Álvarez-Méndez 2001), es uno de los principales recursos pedagógicos utilizados en el proceso formal de educación de los ciudadanos. Según la Federación de Editores Españoles ANELE (2013) el 81,3\% de los docentes reconocen emplear bastante o mucho el libro de texto en el aula; además, $71.9 \%$ de las familias lo consideran imprescindible en la educación de sus hijos.

Como señalan Occelli y Valeiras (2013), la forma en que los libros de texto presentan el contenido está directamente relacionada con cuestiones culturales, políticas y económicas. En el contexto sociopolítico, por ejemplo, desde hace mucho tiempo se entiende que el discurso presentado en los libros de texto puede contener ideologías capaces de influir en el comportamiento de las personas, y en una escala más grande, alimentar conflictos entre naciones (UNESCO 2010).

La falta de un debate más amplio entre la sociedad y los gobiernos, en particular cuando estos presentan reformas educativas que cambian la estructura escolar, promueve un distanciamiento entre lo que la administración propone y la práctica de los docentes, implicando la existencia de dos currículos distintos: el que los documentos prevén y el que los profesores implementan en la realidad de su aula (Rodríguez-Moreno, Pro-Bueno y MolinaJaén 2018). Los docentes acaban buscando en los recursos disponibles una orientación para la implementación del currículum, en particular en el libro de texto. En ese sentido, LópezValentín y Guerra-Ramos (2013) sugieren que los libros de texto son, para los profesores, la interpretación del currículum oficial más cercana de su práctica.

Ortiz-Revilla, Greca y Adúriz-Bravo (2018) puntualizan que las orientaciones educativas actuales de varios países sugieren que los ciudadanos del nuevo milenio deben, a lo largo de su educación formal, desarrollar competencias compatibles con los retos del siglo XXI, directamente vinculadas al desenvolvimiento de la ciudadanía y al desarrollo sostenible mundial. En el contexto de las Ciencias de la Naturaleza hay un consenso de que su enseñanza abarca el conocimiento no sólo de las leyes que rigen los fenómenos de la naturaleza, sino también las diversas maneras como los científicos trabajan (National Research Council 1996; 2000). Para el desarrollo de la competencia científica, entendida como la capacidad para emplear el conocimiento científico, identificar preguntas y obtener conclusiones basadas en pruebas, con el fin de comprender y ayudar a tomar decisiones sobre el mundo natural y los cambios que la actividad humana produce en él (PISA 2006), es importante que el profesor haga uso de metodologías centradas en la figura del estudiante y que estimule el desarrollo de habilidades relacionadas con la práctica científica (Franco-Mariscal, 2015). La metodología de la indagación y el diseño ingenieril destacan en este escenario, incentivando el desarrollo de la competencia científica (Greca y Meneses-Villagrá 2018).

El presente trabajo analiza las actividades de aprendizaje incluidas en los libros de texto de Ciencias de la Naturaleza de la Educación primaria de tres editoriales de España importantes por su ranking de ventas, con el objetivo de identificar si estimulan en los estudiantes el desarrollo de las capacidades y la construcción de conocimientos en contextos de su interés, para ser competentes científicamente. Concretamente buscamos responder a la siguiente pregunta de investigación, ¿en qué medida los libros de texto de Ciencias Naturales de la Educación Primaria han incorporado actividades de aprendizaje que permitan desarrollar la competencia científica y en particular actividades que requieran utilizar procesos científicos e ingenieriles indagatorios? 
Inferir si las actividades de aprendizaje propuestas en los libros de texto promueven la competencia científica es complejo. Entendiendo que esta se demuestra en la acción (Sanmartí y Márquez, 2017), es decir, en un "saber hacer", en el análisis realizado de las actividades hemos puesto el énfasis en si contemplan o impulsan principalmente las dimensiones: metodológica de la competencia científica (Cañal 2012) -relacionada con el conocimiento y uso de procesos necesarios para abordar el estudio de los fenómenos desde una perspectiva científica, la búsqueda y selección de información relevante y la producción y valoración de conclusiones y argumentaciones- e integrada-que implica el establecimiento de relaciones entre distintas capacidades científicas-, por ejemplo, con el uso de la metodología de la indagación y el diseño ingenieril.

En consecuencia, analizar si los libros de texto de ciencias naturales actuales han incorporado actividades que estimulen la competencia científica, como son las de carácter indagatorio integrado, y comparar las mismas con las propuestas en los libros de hace dos décadas, creemos que es una pregunta de investigación pertinente.

\section{Fundamentación teórica}

\section{Análisis de libros de texto}

Occelli y Valeiras (2013) clasifican las investigaciones sobre los libros de texto de ciencias en cuatro categorías: artículos centrados en el contenido científico, artículos centrados en el contenido didáctico, artículos de validaciones metodológicas y artículos de revisiones teóricas. Un estudio centrado en un contenido científico es, por ejemplo, el de Martin-Gámez, PrietoRuz y Jiménez-López (2013) que realizan un análisis cualitativo de los libros de texto relacionados con la problemática energética. Un trabajo sobre análisis de contenido didáctico es el de Moreno-Martínez y Calvo-Pascual (2019) que analizan cómo los libros de texto de educación secundaria presentan la historia de la química. Park y Lavonen (2013) analizan dos libros de texto de ESO, que utilizan el concepto de indagación para presentar los contenidos científicos.

Específicamente considerando el análisis de las actividades de aprendizaje contenidas en los libros de texto, Martínez-Losada y García-Barros (2003) presentan un estudio de los objetivos y procedimientos adoptados en los libros de ciencias utilizados en España. Concluyen que, en su mayoría, los procedimientos propuestos en las actividades de aprendizaje son de bajo nivel de exigencia, centrados en la adquisición de conocimientos de tipo descriptivo y con poco impacto para un aprendizaje significativo; además los autores señalan que no hay actividades de planificación y pocas de interpretación. Aunque anterior a la incorporación de la concepción educativa competencial en el currículo oficial español, este estudio puede ser un referente con el que comparar las pretensiones de las actividades de aprendizaje propuestas en los libros actuales, particularmente en el desarrollo de capacidades procedimentales y en la construcción del conocimiento en contextos de interés, para que ser científicamente competente.

Una década después, López-Valentín y Guerra-Ramos (2013) utilizan los indicadores propuestos por Martínez-Losada y García-Barros (2003), y tratan de valorar cualitativa y cuantitativamente los objetivos de enseñanza, los contenidos procedimentales y el potencial de promoción del aprendizaje que presentan dos libros de texto de Ciencias Naturales de educación primaria en México, ahora en un contexto de desarrollo de competencias, en particular, la competencia científica, entendida como el "desarrollo de habilidades de pensamiento científico y sus niveles de representación e interpretación acerca de los fenómenos y procesos naturales" (SEP, 2009). 
Más recientemente, Laya-Iglesias y Martínez-Losada (2019) analizan el tipo de capacidades que dan ocasión de desarrollar las actividades de aprendizaje propuestas en libros de sexto curso de primaria en la Comunidad Autónoma de Galicia, específicamente examinando el bloque "el ser humano y la salud". Al considerar que la competencia científica está relacionada con el desarrollo del conocimiento y de las actitudes, los autores delimitan el problema de investigación concretamente a la identificación y valoración de habilidades específicas de cuatro tipos: a) relacionadas con el conocimiento específico en sí; b) relacionadas con el uso del conocimiento en situaciones concretas; c) relacionadas con los procesos científicos y d) relacionadas con el uso de evidencias científicas. Los resultados obtenidos indican que no hubo mucha evolución respecto a los estudios anteriores, al menos respecto al bloque analizado en las actividades de aprendizaje, siendo la mayoría de las actividades centradas en el dominio del conocimiento teórico previamente expuesto.

\section{Las actividades indagatorias y el desarrollo de la competencia científica}

En el currículo básico español de la educación primaria (BOE 2014) se establece que a través del área de ciencias de la naturaleza hay que proporcionar a los estudiantes las bases de una formación científica que les ayude a desarrollar las competencias necesarias para desenvolverse en una realidad cambiante cada vez más científica y tecnológica. Una de estas competencias es la científica (PISA 2006) que tratamos en este estudio y que de acuerdo con Cañal (2012) tiene cuatro dimensiones: conceptual, metodológica, actitudinal e integrada. Según PISA (2006) un individuo con competencia científica reúne capacidades para utilizar el conocimiento científico con los siguientes fines: i) Describir, explicar y predecir fenómenos naturales; ii) Comprender los rasgos característicos de la ciencia; iii) Identificar, formular e investigar problemas e hipótesis; iv) Utilizar pruebas, documentarse, argumentar y tomar decisiones personales y sociales sobre el mundo natural y los cambios que la actividad humana genera en él y v) mostrar disposición para implicarse en asuntos relacionados con la tecnociencia y comprometerse con las ideas de la ciencia como un ciudadano reflexivo.

En este sentido, para el despliegue de la competencia científica, es conveniente que los estudiantes desarrollen capacidades de investigación en el contexto escolar, en particular ampliando aquellas asociadas con la dimensión metodológica (Franco-Mariscal, 2015). Considerando la importancia del libro de texto como herramienta educativa, para trasladar al trabajo de aula el desarrollo competencial científico en sus diversas dimensiones, es necesario proponer a los estudiantes, dentro de las actividades de aprendizaje, intervenciones que contemplen todas las dimensiones (Rodríguez-Moreno, Pro-Bueno y Molina-Jaén 2018). Por ello, es importante analizar en qué medida las actividades propuestas en los libros de texto contemplan las dimensiones que conforman la competencia científica, especialmente la metodológica, que permite el desarrollo de las principales estrategias de la metodología científica, tales como la capacidad de formular preguntas, identificar el problema, formular hipótesis, planificar y realizar actividades, observar, recoger y organizar la información relevante, sistematizar y analizar los resultados, sacar conclusiones y comunicarlas, trabajando de forma cooperativa y haciendo uso de forma adecuada de los materiales y herramientas (BOE 2014).

Al igual que Greca y Meneses (2018) creemos que la práctica constante en el aula de procesos indagatorios -científicos e ingenieriles- impulsa en buen grado el desarrollo de la competencia científica en todas sus dimensiones. Según Harlen (1998) una actividad indagatoria es una "actividad más o menos abierta que introduce al alumno en el desarrollo de una serie de procedimientos asociados a la resolución de problemas, es decir, pequeñas investigaciones". A través de las actividades de indagación, el estudiante va construyendo significativamente su conocimiento del mundo natural, a la vez que comienza a entender el proceso de hacer ciencia 
(Greca, Díez-Ojeda y Meneses-Villagrá 2017). Así mismo, el proceso de aprendizaje basado en la indagación, con actividades estratégicamente secuenciadas, es coherente con las teorías socioconstructivistas de aprendizaje, ya que tiene en cuenta los conocimientos previos del aprendiz, promueve la interacción sociocultural y el conflicto cognitivo (Pedrinaceli, 2012; Meneses-Villagrá y Caballero-Sahelices 2017).

Aunque existen diferentes enfoques para el desarrollo de la metodología indagatoria en la enseñanza, algunas etapas (con más o menos énfasis) deben estar presentes: plantear un problema susceptible de investigación a partir de una situación contextualizada, emitir hipótesis, planificar y realizar actividades para contrastar las hipótesis, recoger e interpretar datos o evidencias, establecer conclusiones y comunicar resultados (Zômpero y Laburú 2011). Durante este proceso se pretende potenciar el desarrollo de competencias relacionadas con la capacidad de resolver problemas (Fraiha, Paschoal, Perez, Tabosa y Silva 2018).

En el presente estudio, analizamos el tipo de actividades que presentan actualmente varios libros de tres editoriales españolas, tratando de identificar las dimensiones de la competencia científica que abordan, y de cuantificar, comparativamente, o cuanto han incorporado del enfoque competencial durante la última década. Para ello, a través de su descripción y siguiendo la clasificación de Martínez-Losada y García-Barros (2003) inferiremos los objetivos que persiguen y las capacidades procedimentales que permiten desarrollar al realizarlas, indicadores que nos permitirán valorar en qué medida contemplan las dimensiones conceptual, metodológica e integrada (Cañal 2012). También se comparan los resultados obtenidos con la literatura, para ver si las actividades de los libros actuales persiguen nuevos objetivos y potencian capacidades más relacionados con la competencia científica. La comparación con un estudio previo a la incorporación de la concepción educativa competencial en el currículo oficial español busca cuantificar, si es posible, el impacto de tales directivas oficiales en la práctica docente, intrínsecamente relacionada con el uso del libro de texto. Por último, se destaca la importancia de incluir actividades indagatorias que permitan a los estudiantes mejorar el desarrollo de estrategias de la metodología científica y la comprensión de la naturaleza de la ciencia.

\section{Método}

\section{Libros de texto analizados}

La muestra de análisis está constituida por cuatro libros de texto de Ciencias de la Naturaleza, pertenecientes al cuarto curso (elegido al azar) de la educación primaria, tres escritos en español y uno en inglés, muy utilizados en centros escolares y comercializados por tres editoriales de España. Los temas considerados fueron 'materia y materiales', 'fuerzas y energía' y 'máquinas' pues los profesores que analizaron las actividades -autores de este estudio- tenían más experiencia en estas temáticas. Cada libro fue identificado por un código formado por un número y una letra, perteneciendo el número a la editorial $(1,2$ o 3) y la letra a la lengua en que se ha escrito el libro (E o I).

\section{Procedimiento de análisis de los libros de texto}

Fueron catalogadas todas las actividades encontradas en los libros de texto en formato papel, no analizándose las actividades de aprendizaje propuestas digitalmente ni las existentes en otros posibles libros extras. Entendemos actividad de aprendizaje como todo aquello que demande del alumnado algún tipo de actividad, ya sea mental o física, cuya finalidad sea promover el aprendizaje de los contenidos curriculares (García 1997). Se utilizó predominantemente una metodología con un enfoque cualitativo, realizando un análisis descriptivo de las 542 actividades (408 en castellano y 134 en inglés) propuestas en los libros, 
atendiendo a dos criterios: en función de los objetivos que persiguen y según los contenidos procedimentales que permiten desarrollar. Se recogen en tablas las frecuencias y porcentajes de actividades que persiguen los objetivos y procedimientos seleccionados. En aquellas actividades que fueron identificados más de un objetivo o procedimiento, solo consideramos aquél que exigía del alumno habilidades cognitivas más elaboradas.

$\mathrm{Al}$ observar que los libros en inglés (3I) y en español (3E) de la editorial 3 contenían actividades de aprendizaje muy similares, elegimos analizar solo los libros en español en la discusión de las tendencias generales, para no duplicar el peso de la editorial 3 respecto a las 1 y 2 . Por lo tanto, el libro en inglés solo se utilizó para identificar diferencias en relación con las actividades de aprendizaje propuestas entre materiales escritos en inglés y en español de una única editorial.

Para catalogar a las actividades según el objetivo que persiguen, adaptamos la clasificación de Martínez y García (2003) incluyendo una categoría más para aquellas actividades que propongan el desarrollo de un proyecto de ingeniería o elaboración de un producto. Las actividades que comúnmente se denominan 'recetas de cocina' fueron clasificadas en la categoría denominada 'desarrollo de técnicas', principalmente de registro o clasificación. Por ejemplo, al estudiar técnicas de separación de mezclas de sustancias, se propone seguir una secuencia de pasos específica para varios métodos de separación; concretamente en el caso del tamizado: i) echar la mezcla en un vaso con agua; ii) retirar las bolitas de corcho con un colador; y iii) verter el agua en otro recipiente (para separar la mezcla corcho blanco y arena). En la Tabla 1 presentamos una breve descripción de los objetivos considerados para clasificar las actividades de aprendizaje propuestas por los libros.

Para catalogar los contenidos procedimentales que se trabajan mediante las actividades propuestas en los libros, readaptamos la clasificación de Martínez y García (2003). Trasladamos el procedimiento concreto 'palabra/frase' del ámbito 'comunicaciones' al de 'organización de la información' incluyéndolo dentro del procedimiento concreto 'descripción simple', ya que entendemos que al realizar una descripción simple utilizando una palabra o frase se pretende organizar la información; por ejemplo, después de presentar un texto explicando lo que es la energía solar y la diferencia entre paneles fotovoltaicos y térmicos, se propone al estudiante la siguiente pregunta: "¿qué forma de energía se aprovecha en los paneles solares térmicos? y ¿en los paneles solares fotovoltaicos?" Esta actividad fue clasificada como organización de la información (descripción simple).

Tabla 1. Análisis descriptivo de los objetivos de las actividades de aprendizaje, según Martínez-Losada y García -Barros (2003a) apud Lópes-Valentín y Guerra-Ramos (2013).

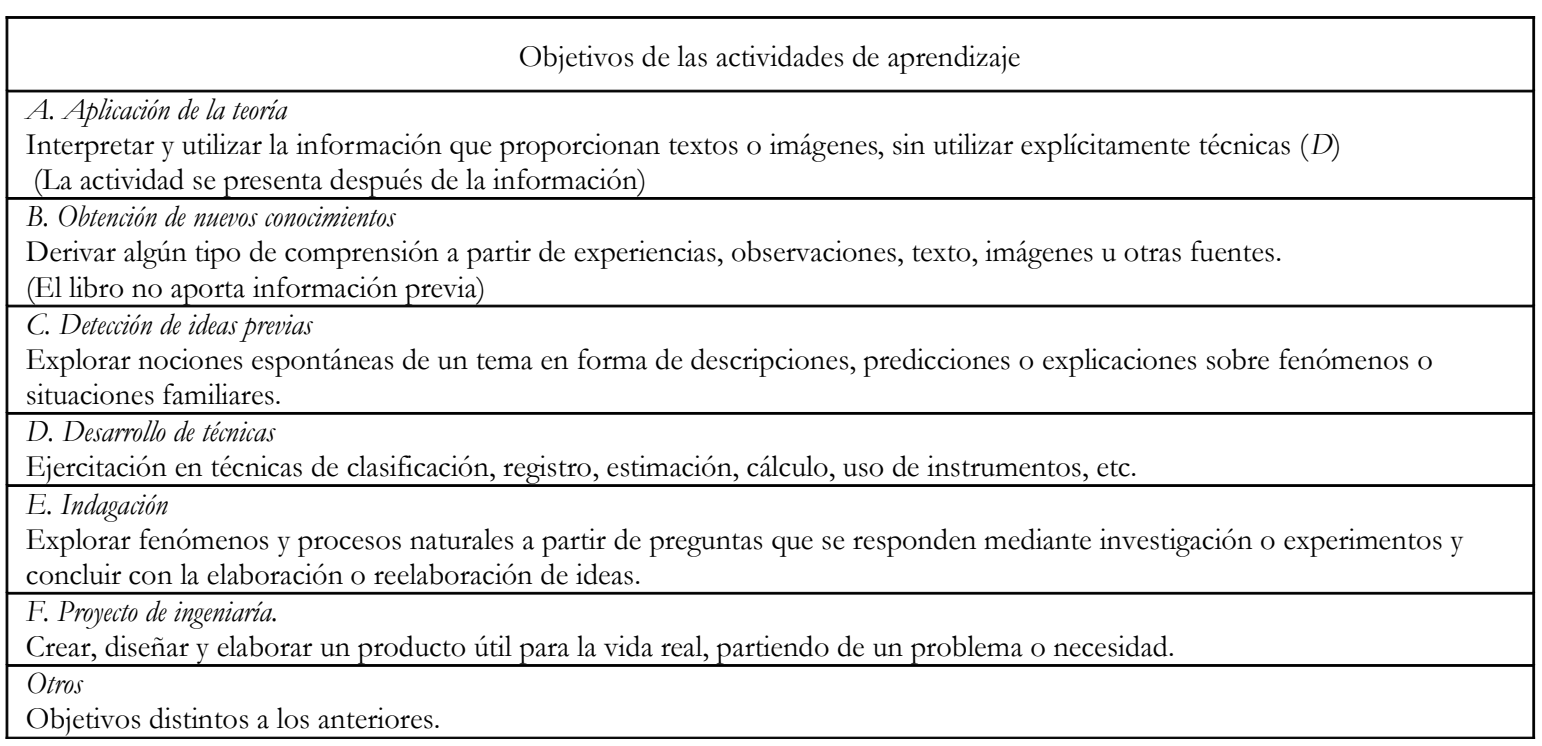


También cambiamos el procedimiento concreto 'tablas y gráficos' del ámbito procedimental 'comunicación' y lo incluimos en el de 'organización de la información', y creamos un nuevo procedimiento 'interpretación de texto y audio' en el ámbito interpretación. Añadimos nuevos procedimientos en el ámbito 'habilidades manipulativas y de cálculo', concretamente los de 'construcción libre' y 'construcción guiada'. Las construcciones guiadas fueron consideradas como aquellas donde el estudiante debe seguir una serie de pasos predeterminados para llegar al resultado final y las construcciones libres no presentan dichos pasos. Por ejemplo, al estudiar los tipos de máquinas dos editoriales proponen la construcción de una grúa; la editorial 3E detalla una construcción guiada (i. hacer la base llenando un envase vacío con arena; ii. hacer el brazo con un tubo largo de cartón; iii. enganchar las partes y meter los carretes de hilo en el tubo; iv. pasar el hilo por los carretes), sin embargo, la editorial 2 plantea al estudiante que diseñe y construya libremente una grúa con los materiales que tenga a su alcance (construcción libre).

Tabla 2. Análisis descriptivo de los contenidos procedimentales de las actividades de aprendizaje, adaptada de Martínez-Losada y García-Barros (2003a).

\begin{tabular}{|c|c|}
\hline Ámbito procedimental & Procedimiento concreto \\
\hline \multirow{3}{*}{ Planificación del proceso } & Emisión de hipótesis \\
\hline & Identificación o control de variables \\
\hline & Diseño de experiencia \\
\hline \multirow{2}{*}{ Observación } & Directa de los objetos presentes \\
\hline & Indirecta de representaciones gráficas \\
\hline Búsqueda de información en otras fuentes & Internet, revistas, otros libros. \\
\hline \multirow{7}{*}{ Organización de la información } & Descripción simple \\
\hline & Identificación de características \\
\hline & Establecimiento de relaciones \\
\hline & Reconocimiento de diferencias y semejanzas \\
\hline & Ordenación \\
\hline & Clasificación \\
\hline & Construcción de tablas o gráficos \\
\hline \multirow{4}{*}{ Comunicación } & Resúmenes o informes \\
\hline & Murales, esquemas o dibujos \\
\hline & Debates al final \\
\hline & Intercambio de ideas, opiniones (hablar) \\
\hline \multirow{4}{*}{ Interpretación } & Textos/Audios \\
\hline & Hechos/fenómenos/situaciones \\
\hline & Datos numéricos o tablas \\
\hline & Gráficos \\
\hline Elaboración de conclusiones & Generalizaciones basadas en las evidencias \\
\hline \multirow{6}{*}{$\begin{array}{l}\text { Destrezas o habilidades manipulativas y de } \\
\text { cálculo }\end{array}$} & Uso sencillo de material \\
\hline & Manejo de instrumentos observación/medida \\
\hline & Recogida de muestras \\
\hline & Construcción o técnica guiada \\
\hline & Construcción o técnica libre/abierta \\
\hline & Cálculos \\
\hline
\end{tabular}

Las actividades propuestas en los libros que formulan preguntas antes de la introducción del contenido conceptual (por ejemplo, un concepto, una clasificación o una ley), las catalogamos como de 'emisión de hipótesis', y aquellas propuestas después de la presentación y discusión del concepto, que también plantean preguntas, en el procedimiento 'interpretación de hechos, fenómenos y situaciones'. Concretamente, en el libro escrito en inglés, fueron encontradas varias actividades que sustituían la interpretación de texto por la interpretación de audios. Por 
ello, en el procedimiento 'interpretación de textos' añadimos también audios. La tabla 2 recoge los procedimientos específicos que hemos seleccionado, dentro de distintos ámbitos procedimentales.

\section{Resultados y su análisis}

Los resultados obtenidos sobre los dos criterios seleccionados y el análisis correspondiente se ofrecen a continuación.

\section{Objetivos que proponen las actividades}

En la tabla 3 se presenta el número de actividades propuestas por los libros escritos en español según el objetivo que persiguen y sus resultados porcentuales comparados con los obtenidos por Martínez-Losada y García-Barros (2003). En general, las actividades con el propósito de fijar contenido específico (aplicación de la teoría y obtención de nuevos conocimientos) son la mayoría $(72 \%)$. Este porcentaje complementa el obtenido por Laya-Iglesias y Martínez-Losada (2019), analizando actividades del tema "El ser humano y la salud" en libros de sexto de primaria, donde señalan que el $80 \%$ de las actividades de aprendizaje están relacionadas con los contenidos conceptuales tratados de manera expositiva.

Tabla 3. Análisis comparativo de los resultados encontrados y los de Martínez-Losada y García-Barros (2003) para los objetivos de las actividades de aprendizaje (solo considerando los libros en castellano)

\begin{tabular}{|c|c|c|c|}
\hline \multirow[b]{2}{*}{ Objetivos } & \multicolumn{2}{|c|}{ Estudio de esta investigación } & \multirow{2}{*}{$\begin{array}{l}\text { Estudio de } \\
\text { Martínez y } \\
\text { García (2003) }\end{array}$} \\
\hline & $\begin{array}{l}\text { Número de } \\
\text { actividades }\end{array}$ & $\begin{array}{c}\text { Porcentaje de } \\
\text { actividades }\end{array}$ & \\
\hline $\begin{array}{l}\text { A. Aplicación de la teoría } \\
\text { Interpretar y utilizar la información que proporcionan textos o } \\
\text { imágenes. } \\
\text { (La actividad se presenta después de la información) }\end{array}$ & 245 & $60 \%$ & $51 \%$ \\
\hline $\begin{array}{l}\text { B. Obtención de nuevos conocimientos } \\
\text { Derivar algún tipo de comprensión a partir de experiencias, } \\
\text { observaciones, texto, imágenes u otras fuentes. } \\
\text { El libro no aporta información previa. }\end{array}$ & 51 & $12 \%$ & $29 \%$ \\
\hline Subtotal I $(A+B)$ & & $72 \%$ & $80 \%$ \\
\hline $\begin{array}{l}\text { C. Detección de ideas previas } \\
\text { Explorar nociones espontáneas de un tema en forma de } \\
\text { descripciones, predicciones o explicaciones sobre fenómenos } \\
\text { o situaciones familiares. }\end{array}$ & 31 & $8 \%$ & $13 \%$ \\
\hline $\begin{array}{l}\text { D. Desarrollo de técnicas } \\
\text { Ejercitación en técnicas de clasificación, registro, estimación, } \\
\text { cálculo, uso de instrumentos, etc. }\end{array}$ & 53 & $13 \%$ & $7 \%$ \\
\hline $\begin{array}{l}\text { E. Indagación } \\
\text { Explorar fenómenos y procesos naturales a partir de preguntas } \\
\text { que se responden mediante investigación o experimentos y } \\
\text { concluir con la elaboración o reelaboración de ideas. }\end{array}$ & 0 & $0 \%$ & $0 \%$ \\
\hline F. Proyecto de Ingeniería & 25 & $6 \%$ & - \\
\hline Subtotal II $(D+E+F)$ & & $19 \%$ & - $7 \%$ \\
\hline $\begin{array}{l}\text { Otros } \\
\text { Objetivos distintos a los anteriores. }\end{array}$ & 3 & $1 \%$ & $0 \%$ \\
\hline Total & 408 & $100 \%$ & $100 \%$ \\
\hline
\end{tabular}

Al analizar la evolución de las actividades, por término medio, las tres editoriales han incrementado un $9 \%$ (del 51\% al 60\%) las actividades de aprendizaje con objetivos 'aplicación de la teoría' respecto al estudio de Martínez y García (2003), y un 6\% (del 7\% al 13\%) las de 'desarrollo de técnicas', esta última presumiblemente más relacionada con el desarrollo de la dimensión metodológica de la competencia científica. Es importante destacar que todos los 
libros escritos en español han incorporado actividades sobre proyectos de ingeniería, sin embargo, estos proyectos no se presentan en el libro en inglés. En cambio, en ninguno de ellos se propone al menos una sola actividad que permita a los estudiantes llevar a cabo un proceso indagatorio completo.

Después de 15 años, las editoriales analizadas han incrementado del 7\% al 19\% las actividades relacionadas con el desarrollo de las dimensiones metodológica e integrada de la competencia científica (desarrollo de técnicas, indagación y proyectos de ingeniería). En cambio, han reducido en un $8 \%$ (del $80 \%$ al $72 \%$ ) las actividades con objetivos centrados en el conocimiento científico teórico (aplicación de teoría y obtención de nuevos conocimientos) -relacionados con la dimensión conceptual de la competencia científica- y en un 5\% (del 13\% al 8\%) las que persiguen objetivos de detección de ideas previas. Estos resultados indican una cierta tendencia a incorporar objetivos alineados con el enfoque competencial, en particular con la dimensiones metodológica e integrada de Cañal (2012).

Entendemos como positiva la presencia en los libros de actividades correctamente enfocadas que estimulen el desarrollo de proyectos de ingeniería. Sin embargo, la ausencia de actividades con objetivos de marcado carácter indagatorio es un dato preocupante, si se considera que los libros de texto son el principal recurso didáctico utilizado por los profesores de Ciencias de la Naturaleza, ya que los estudiantes tendrán pocas oportunidades para llevar a cabo indagaciones científicas completas, donde puedan identificar problemas y dar una respuesta fundamentada en evidencias obtenidas utilizando técnicas y procesos científicos, que les permita desarrollar todas las dimensiones de la competencia científica demandada en los currículos (Franco-Mariscal 2015; Rodríguez-Moreno, Pro-Bueno y Molina-Jaén 2018; Greca y Meneses-Villagrá 2018).

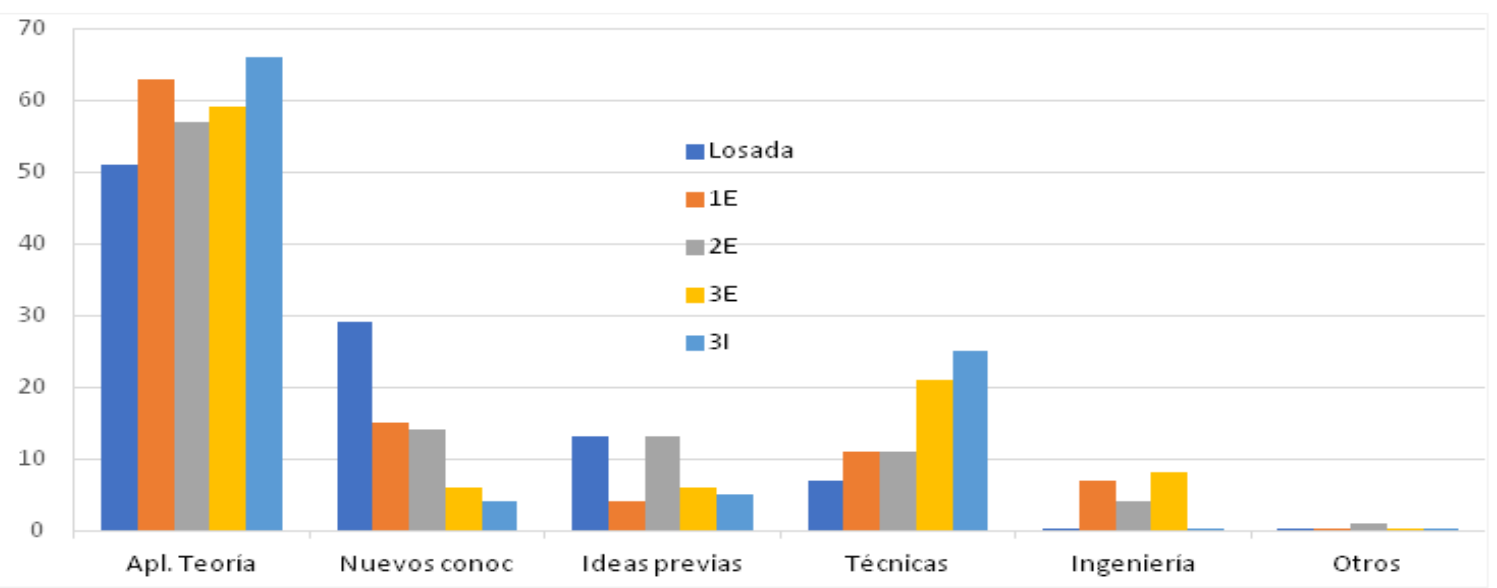

Figura 1. Análisis comparativo de los objetivos de las actividades propuestas en libros de distintas editoriales.

En la figura 1 presentamos unos resultados más detallados de cómo se distribuyen los objetivos por editorial. Respecto al objetivo 'aplicación de la teoría', la editorial $1 \mathrm{E}$ propone bastantes actividades que requieren utilizar directamente la información que se proporciona en el texto (por ejemplo, en un párrafo del texto se dice que "la rueda ...." y en una de las actividades se hace la pregunta, ¿qué es la rueda?); sin embargo, las otras dos editoriales, $2 \mathrm{E}$ y $3 \mathrm{E}$, presentan prioritariamente actividades de interpretación de lo que fue estudiado, con el objetivo de aplicar la teoría (por ejemplo el editorial 2E, al trabajar cómo la luz se desplaza, presenta como actividad la siguiente pregunta: ¿se vería igual la sombra si usáramos un vaso de cristal en vez de madera? ¿y si la luz no se transmitiera en línea recta?). No hay diferencia entre el libro en inglés y el libro en español de la editorial 3, aunque el libro en inglés sustituye parte de las actividades de lectura e interpretación de texto por actividades de interpretación de 
audio. Considerando todas las editoriales, una media del 73\% de las actividades de aplicación de la teoría busca estimular en los estudiantes la interpretación de la información que figura en el texto.

Sobre las actividades con el objetivo 'obtención de nuevos conocimientos', las editoriales 1E y $2 \mathrm{E}$ proponen en una tercera parte de las mismas el uso de Tecnologías de la Información y de la Comunicación (TIC); sin embargo, la editorial 3, tanto el libro en inglés como el de español, apenas plantea actividades que requieran la utilización de las TIC. Resulta sorprendente la disminución de actividades, en un 5\%, focalizadas a la detección de ideas previas en los libros 1E, 3E y 3I respecto al estudio de Losada y Barros de hace 15 años, cuando las teorías constructivistas de aprendizaje siguen considerándose en la implementación del proceso de enseñanza y aprendizaje, demandando la necesidad de partir de las concepciones de los estudiantes y haciéndolas evolucionar hacia las científicas (Pedrinaceli, 2012; Meneses-Villagrá y Caballero-Sahelices 2017).

Con respecto a las actividades con el objetivo 'desarrollo de técnicas', la gran mayoría de las actividades de las tres editoriales están centradas en que los estudiantes desarrollen principalmente el aprendizaje de técnicas de registro y de clasificación, no habiendo tampoco diferencias significativas entre los libros en inglés y español. Solo la editorial $1 \mathrm{E}$ introduce actividades de desarrollo de técnicas de uso de instrumentos.

Es importante señalar que el único material que no contiene ninguna actividad con el objetivo de desarrollo de proyecto de ingeniería es el libro escrito en inglés (figura 1). Anghel, Cabrales y Carro (2013) consideran que enseñar ciencias en un contexto bilingüe (español/inglés) implica peores resultados en términos de alfabetización científica de los estudiantes. El aprendizaje de las ciencias en estos casos es memorístico y se centra en el dominio de los términos aislados, sin trabajar adecuadamente en todas las dimensiones de la competencia científica, en particular la metodológica (Cristóbal-Aragón y Greca 2018). La falta de actividades de aprendizaje que integren todas las dimensiones de la competencia científica, como el desarrollo de proyectos de ingeniería en el libro de inglés (3I), está relacionada con el comportamiento anterior.

\section{Contenidos procedimentales que abordan las actividades}

En este apartado se analizan los distintos contenidos procedimentales que persiguen las actividades propuestas por los libros de las tres editoriales seleccionadas comparándolos con los obtenidos en el análisis realizado por Martínez y García (2003a). La tabla 4 presenta el número de actividades de los tres libros escritos en castellano y los porcentajes medios de las capacidades que pueden potenciarse con las actividades que proponen.

Tabla 4. Análisis comparativo de los resultados y Martínez y García (2003) para los contenidos procedimentales de las actividades de aprendizaje.

\begin{tabular}{|c|c|c|c|}
\hline \multirow[b]{2}{*}{ Procedimiento } & \multicolumn{2}{|c|}{ Estudio de esta investigación } & \multirow{2}{*}{$\begin{array}{l}\text { Estudio Losada y Barros } \\
\text { (temas de Primaria: materia, } \\
\text { animales y vegetables) }\end{array}$} \\
\hline & $\begin{array}{l}\text { Número de } \\
\text { actividades }\end{array}$ & $\begin{array}{c}\text { Porcentaje de } \\
\text { actividades }\end{array}$ & \\
\hline Planificación & 17 & $4 \%$ & $1 \%$ \\
\hline Observación & 8 & $2 \%$ & $15 \%$ \\
\hline Búsqueda de información & 23 & $6 \%$ & $2 \%$ \\
\hline Organización de la información & 169 & $41 \%$ & $33 \%$ \\
\hline Comunicación & 67 & $16 \%$ & $40 \%$ \\
\hline Interpretación & 105 & $26 \%$ & $6 \%$ \\
\hline Conclusiones & 0 & $0 \%$ & $0 \%$ \\
\hline Destrezas manipulativas y de cálculo & 19 & $5 \%$ & $3 \%$ \\
\hline Total & 408 & $100 \%$ & $100 \%$ \\
\hline
\end{tabular}


Las actividades propuestas en los libros que permitan realizar planificaciones de procesos son muy escasas, apenas representan un 4\%, un 3\% más que en el estudio de Losada y Barros. Ejemplos de actividades encontradas con este contenido procedimental son: "fijate en el tamaño de los bloques que forman la pirámide, ¿cómo crees que los colocaron?" o "piensa en otra forma diferente de separar las sustancias de esta mezcla". Destacamos que en ninguna de estas actividades de planificación se plantea pensar en diseños experimentales donde sea necesario controlar variables.

Las actividades focalizadas a organizar información son las más numerosas, pasando del 33\% que figura en el estudio de Martínez-Losada y García-Barros a un $41 \%$ en el actual, resultado similar al encontrado por Laya-Iglesias y Martínez-Losada (2019). Es decir, aproximadamente cuatro de diez actividades propuestas en los libros de las editoriales actuales fomentan el desarrollo de distintas técnicas para organizar la información. Dentro de estas, el 31,1\% son de clasificación y un $44 \%$ de descripción simple, es decir tres de cada cuatro actividades demandan realizar clasificaciones (por ejemplo, "clasifica los materiales en conductores o aislantes") o describir algún hecho o fenómeno (por ejemplo, "describe el proceso de decantación"). Solo un 4,1\% de las actividades piden la realización de tablas y ninguna la construcción de gráficos, lo cual es sorprendente, pues es una de las habilidades necesarias para establecer conclusiones y comunicar los resultados. Pedrinaci (2012) considera que para argumentar científicamente es necesario un tratamiento articulado entre el conocimiento teórico, la capacidad de seleccionar lo que es relevante y la destreza para organizar las informaciones

El siguiente grupo de actividades que más proponen los libros, son las de interpretación, representando aproximadamente la cuarta parte del total, concretamente un $26 \%$ frente al solo $6 \%$ del estudio de Martínez y García, es decir, los libros actuales promueven cuatro veces más la capacidad interpretativa en los estudiantes. De estas, la mayoría $(73,9 \%)$ son actividades que solicitan la interpretación de situaciones y hechos, por ejemplo, “¿por qué las energías solar, eólica e hidráulica suelen denominarse energías limpias?". Destacamos que solamente se encontró una actividad de interpretación de datos y cuatro de interpretación de gráficos. Al no trabajar con los estudiantes, la capacidad de construir e interpretar datos y gráficos afecta a la capacidad de argumentar científicamente (Pedrinaci 2012). Así mismo, detectamos una pequeña diferencia entre los libros en castellano e inglés de la editorial 3: mientras que en castellano el 90\% de las actividades se refieren a interpretar fenómenos y el 10\% a interpretar textos, en inglés se proponen menos actividades para interpretar fenómenos (el 67\%) y más para interpretar textos (el 32\%).

Las actividades encuadradas como comunicación representan un 16\% del total, disminuyendo en más de la mitad respecto al estudio de hace quince años, que representaban el $40 \%$. Dentro de esta categoría, los libros presentan casi un $42 \%$ de actividades solicitando la elaboración de murales, esquemas o dibujos, casi un 39\% son para intercambiar ideas y opiniones y la restante quinta parte demanda la realización de resúmenes o informes escritos.

Las actividades que permiten el desarrollo de destrezas o habilidades manipulativas y de cálculo solamente representan un 5\% del total analizado, de las cuáles la décima parte (concretamente tres) son actividades que solicitan la construcción, sin ninguna guía, de un producto (por ejemplo, una grúa o una catapulta).

Destacamos una disminución importante de actividades con contenido procedimental de 'comunicación' (del 40\% al 16\%) y el aumento de las de 'organización de la información' (del $33 \%$ al $41 \%$ ) entre el estudio de Martínez y García y el presente. Aunque un porcentaje de esta 
importante reducción de las actividades que promueven la 'comunicación' se justifica por el cambio que realizamos en la estructura de la tabla (recordamos que nosotros incluimos el procedimiento concreto 'palabra/frase' del ámbito procedimental 'comunicaciones' en el de 'descripción simple' del ámbito de 'organización de la información'), nos preocupa que las editoriales en sus libros de texto fomenten la capacidad de comunicación menos que hace quince años. Laya-Iglesias y Martínez-Losada, (2019) también puntúan la presencia reducida de actividades de elaboración de conclusiones y argumentación.

Por último, hemos detectado un pequeño incremento del $3 \%$ en los contenidos procedimentales de planificación y del $2 \%$ en desarrollo de destrezas manipulativas y de cálculo, e inexistencia de actividades que demanden establecer conclusiones. Estas capacidades procedimentales y otras fundamentales para desarrollar la competencia científica, como la identificación de problemas, poco consideradas en los libros de texto se impulsarían si en estos se plantearan adecuadamente actividades cuya finalidad fuera realizar distintos tipos de indagaciones para aplicar los procesos que caracterizan a las ciencias y al método de la investigación científica. (Franco-Mariscal 2015; Rodríguez-Moreno, Pro-Bueno y Molina-Jaén 2018; Greca y Meneses-Villagrá 2018).

En la figura 2 presentamos un análisis más detallado de cómo se distribuyeron los contenidos procedimentales por editorial. Se observa que la editorial 1 presenta un porcentaje muy grande de actividades de organización de la información, al comparándola con las otras editoriales, a costa de disminuir las de contenido procedimental de comunicación, planificación y interpretación. La mayoría de sus actividades están asociadas con el objetivo de 'aplicación de la teoría', específicamente con el uso directo de la información que se proporciona en el texto. Las editoriales 2 y 3 proponen actividades bastante semejantes, excepto en las de observación que la editorial 3 no presenta ninguna, ni en su libro en español 3E ni en el de inglés $3 \mathrm{I}$.

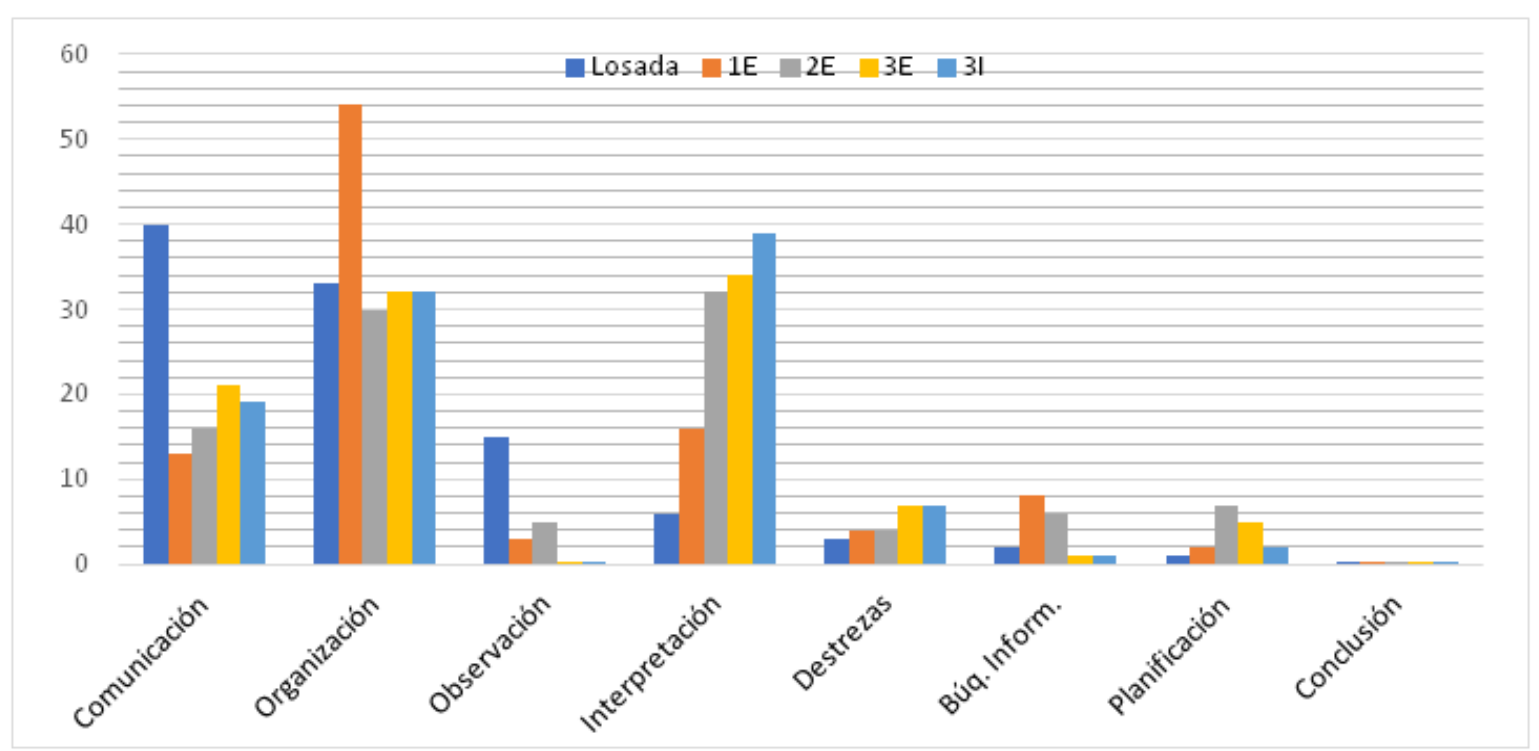

Figura 2. Análisis comparativo de los contenidos procedimentales abordados por las actividades propuestas en libros de distintas editoriales

\section{Implicaciones educativas}

En el análisis anterior hemos detectado que los libros de texto mayoritariamente contemplan actividades con la finalidad de aplicar el conocimiento científico a través de textos o imágenes, para facilitar principalmente el desarrollo de las capacidades de organización de la información, interpretación y comunicación. Sin embargo, detectamos que faltan otras 
capacidades necesarias para desarrollar la competencia científica, tal y como es entendida en PISA 2006, como son las de identificar cuestiones científicas, extraer conclusiones basadas en pruebas sobre temas tecnocientíficos, comprender los procesos de desarrollo de la ciencia y tomar conciencia de las formas como la ciencia y tecnología moldean nuestro entorno material, intelectual y cultural.

Para ello, consideramos que los libros deben proponer otro tipo de actividades para que los estudiantes apliquen los procesos que caracterizan la ciencia dando respuestas a problemas surgidos en un contexto de situaciones reales y vividas. A continuación, a modo de ejemplo, proponemos y explicamos dos tipos de actividades que deben ser contempladas mucho más en los libros de ciencias y que contemplan las dimensiones de la competencia científica: una indagación científica (propuesta por los autores) y un proyecto de ingeniería (Solas-Ruíz 2018), que se han llevado a cabo con estudiantes de cuarto año de primaria del C.E.I.P. Miguel Delibes, Burgos.

\section{Indagación científica}

Presentamos el estudio de las mezclas utilizando la metodología didáctica indagatoria, para que los docentes con las adaptaciones que crean necesarias puedan llevarlo a cabo en su aula de ciencias. Nosotros concebimos el proceso indagatorio como un ciclo formado por cinco etapas: i) situación problematizadora, ii) problema de investigación e hipótesis, iii) diseño experimental, iv) resultados y conclusiones, y v) afianzamiento y comunicación. Estas etapas son flexibles y dependiendo de las circunstancias se pueden intercambiar, añadir otras o volver de una más avanzada a otra más inicial o al revés.

Una situación problematizadora puede presentarse a través de un cuento, un video, un viaje, experimentos sorprendentes, etc. En esta indagación, que puede consultarse en la web http://www.webciencia.es/index.php/articulos/242-vacaciones-en-barcelona-fases-e-informacion, los estudiantes leen el siguiente relato: "En las últimas vacaciones Julia fue con sus padres a visitar a sus primos que viven en un pueblo de Barcelona. Cuando llegaron hacia mucho calor y habia muchos turistas visitando el hermoso pueblo costero. Debido al exceso de visitantes, el Ayuntamiento detectó que la demanda de agua potable era muy alta y se necesitaba poner en funcionamiento la planta desalinizadora que disponía el municipio para producir agua potable a través del agua del mar".

Tras analizar la situación problematizadora, el profesor promueve un intercambio de ideas entre los estudiantes, solicitando preguntas científicas y respuestas tentativas a las mismas; los estudiantes son capaces de identificar preguntas, por ejemplo: "¿qué sustancias tiene el agua del mar que cogemos en la playa con un cubo?", "¿las podemos ver o detectar todas ellas?", “¿las podemos separar?". Al contestar estas preguntas, los estudiantes expresan sus ideas previas, por ejemplo: "cuando añadimos algas, hojas o arena al agua, estas sustancias las vemos y podemos separarlas con las manos, aunque la arena es más difícil", "el agua del mar está salada, ha desaparecido la sal porque no se ve", "no podemos separar la sal del agua", etc.

Antes de comenzar la segunda etapa, el profesor dirige el debate con los estudiantes para proponer el(los) problema(s) de investigación que hay que abordar: ¿qué ocurre cuando juntamos dos o más sustancias sólidas o líquidas? y ¿cómo podemos separarlas? Para responder al primer problema de investigación, los estudiantes emiten sus hipótesis:

- Si mezclamos sustancias en estado sólido todas ellas siguen existiendo porque las seguimos viendo y diferenciando.

- Si mezclamos sustancias en estado líquido o con otras en estado sólido, dependiendo de cuáles sean, pueden distinguirse o no, pero las dos sustancias siguen existiendo.

En la tercera etapa, los estudiantes deben debatir en grupo y presentar propuestas de diseños 
experimentales para testar las hipótesis planteadas. Proponen mezclar distintas sustancias en estado sólido y líquido en vasos de plástico transparente. Antes de proceder a las mezclas se les propone que escriban en una tabla sus predicciones sobre lo que creen que va a ocurrir cuando se mezclan las sustancias que deseen o diga el profesor, por ejemplo, piedrecitas y arena, arroz y agua, trocitos de hierro y arena, sal y agua, aceite y agua, alcohol y agua, entre otras.

Tras ejecutar los experimentos, los estudiantes deben recoger en otra tabla lo que observan cuando se mezclan las sustancias, ayudándose de un dibujo, y lo comparan con sus predicciones; se insiste en que digan si se pueden identificar o no las dos sustancias, si siguen existiendo o si se han transformado en otras. Estos dados serán utilizados para contrastar las hipótesis planteadas. El profesor presenta los términos, homogéneo y heterogéneo, y los grupos clasifican las mezclas realizadas.

En la última etapa de afianzamiento y comunicación, los estudiantes clasifican nuevas mezclas, elaboran un mapa conceptual, reflexionan sobre el proceso que han seguido para contestar al problema y lo comunican al resto de compañeros.

La enseñanza continúa proponiendo el profesor indagar sobre distintos métodos para separar las sustancias mezcladas, iniciándose un nuevo proceso investigativo para contestar al segundo problema.

\section{Método Ingenieril}

El método ingenieril se diferencia de una indagación porque, en lugar de ejercer el estudiante como un científico para resolver un problema susceptible de investigación, debe proceder como un ingeniero, proyectando y elaborando un producto que responda al problema planteado. La validación y utilidad del producto desarrollado están vinculadas a los condicionantes establecidos, como: costo, material disponible, público, funciones ...

Para desarrollar el método ingenieril en el aula de educación primaria, contemplamos también cinco etapas totalmente flexibles: i) definición del problema, ii) búsqueda de información pertinente, iii) planificación y diseño del prototipo, iv) prueba del prototipo, v) mejora del producto. A modo de ejemplo, resumimos la secuencia didáctica propuesta por Solas (2018) donde se trabajan conceptos de mecánica clásica con estudiantes de últimos niveles de educación primaria por medio de la construcción de una catapulta.

Para la introducción al problema, Solas presenta a los estudiantes la imagen de una catapulta y un video sobre la historia de las catapultas, proponiéndoles la construcción de una que permita lanzar objetos para derribar un muñeco situado a una distancia determinada. A continuación, los estudiantes deben buscar informaciones sobre su funcionamiento, su evolución, los conceptos científicos implicados, las variables que influyen en la precisión y alcance de las piedras, etc. Se finaliza esta etapa con la emisión de hipótesis sobre las relaciones entre diversas variables.

Con las informaciones tomadas, los estudiantes planifican y diseñan algunos prototipos para testar la dependencia de la precisión y alcance con las variables señaladas en las hipótesis, como: ángulo de disparo, longitud del brazo, masa del proyectil. 
Tabla 5. Ejemplo de tabla para recoger los resultados de tres prototipos. Adaptada de Ruiz (2018).

\begin{tabular}{|l|c|c|c|c|c|c|}
\hline & \multicolumn{2}{|c|}{ Diseño A } & \multicolumn{2}{c|}{ Diseño B } & \multicolumn{2}{c|}{ Diseño C } \\
\cline { 2 - 7 } & $\begin{array}{c}\text { Angulo de } \\
\text { disparo (grados) }\end{array}$ & $\begin{array}{c}\text { Alcance } \\
(\mathrm{cm})\end{array}$ & $\begin{array}{c}\text { Longitud del } \\
\text { brazo }(\mathrm{cm})\end{array}$ & $\begin{array}{c}\text { Alcance } \\
(\mathrm{cm})\end{array}$ & $\begin{array}{c}\text { Masa del } \\
\text { proyectil (g) }\end{array}$ & $\begin{array}{c}\text { Alcance } \\
(\mathrm{cm})\end{array}$ \\
\hline Lanzamiento 1 & & & & & & \\
\hline Lanzamiento 2 & & & & & & \\
\hline Lanzamiento 3, 4, 5,.. & & & & & & \\
\hline
\end{tabular}

A continuación, los distintos grupos de estudiantes realizan el diseño de sus catapultas, construyen los prototipos y los prueban, se miden los datos obtenidos en los distintos lanzamientos, se organizan para su interpretación (en la tabla 5) y se discuten en el aula.

Teniendo en cuenta las ventajas e inconvenientes de los distintos diseños y prototipos de catapultas ensayados, los datos medios y de precisión de alcance recogidos, así como los condicionantes establecidos (costo máximo, materiales, estética, etc.), los grupos de estudiantes mejoran sus prototipos para crear y construir su producto final.

\section{Conclusiones}

El currículo básico de la educación primaria (BOE 2014) establece que a través del área de Ciencias de la Naturaleza los estudiantes deben iniciarse en el desarrollo de las principales estrategias de la metodología científica. Hemos comprobado que los libros de esta área, de tres editoriales españolas, proponen muchas actividades aisladas que permiten el desarrollo de algunas de las capacidades que se requieren para 'hacer ciencia', principalmente las de búsqueda y organización de información, interpretación y comunicación, en menor medida las de planificación, observación y destrezas manipulativas y de cálculo y apenas se potencian capacidades como formular preguntas, identificar problemas, diseñar experimentos para contrastar hipótesis y predicciones, o plantear explicaciones a partir de las evidencias obtenidas. También hemos detectado que apenas se presentan actividades más amplias que permitan ejecutar procesos indagatorios científicos e ingenieriles completos en un contexto de situaciones del entorno de los estudiantes. Resultados similares a los encontrados por LayaIglesias y Martínez-Losada (2019) en el tema Ser humano y la Salud. También los maestros de educación primaria consideran que las editoriales suelen estar distantes a lo establecido en los programas oficiales respecto al desarrollo de la competencia científica (Pro-Bueno y MolinaJaén 2018). Este tipo de actividades son necesarias pues su enfoque pedagógico potencia el desarrollo de la competencia científica, ya que tiene en cuenta la forma en que los estudiantes construyen progresivamente las ideas científicas, desde el desarrollo de las habilidades que forman parte de la dinámica científica como: formular preguntas desde los intereses y motivaciones de los estudiantes, plantear y ejecutar una metodología de investigación, analizar los resultados, concluir y abrir espacios de discusión y socialización (IAP, 2010), potenciando el desarrollo de todas las dimensiones de la competencia científica (Franco-Mariscal 2015; Rodríguez-Moreno, Pro-Bueno y Molina-Jaén 2018; Greca y Meneses-Villagrá 2018).

El análisis comparativo sobre la finalidad de las actividades propuestas por libros de hace quince años (Martínez y García 2003) y los actuales muestran una pequeña tendencia de cambio, con la incorporación de más actividades con objetivos relacionadas con el desarrollo de técnicas -principalmente de clasificación, registro y cálculo- y algunos proyectos de ingeniería, aunque con pautas demasiado detalladas, como si fueran recetas de cocina. Estos resultados son consistentes con los encontrados en estudios similares (Rodríguez-Moreno, Pro-Bueno y Molina-Jaén 2018; Laya-Iglesias y Martínez-Losada 2019). Aunque se puede observar pequeños cambios en las actividades de aprendizaje, ninguno de los libros propone la 
realización de indagaciones sobre situaciones contextualizadas, donde los estudiantes puedan utilizar procesos análogos a los empleados por los científicos. Tanto el desarrollo de procesos indagatorios como proyectos de ingeniería, como los mostrados en el apartado anterior, son muy útiles para desarrollar la competencia científica (PISA, 2006).

La ciencia y la tecnología constituyen una empresa humana, ejerciendo una notable influencia en la sociedad en la que vivimos y en nosotros mismos. Debemos procurar que los estudiantes se impliquen en temas tecnocientíficos y a comprometerse con las ideas de la ciencia como un ciudadano reflexivo (Fraiha, Paschoal, Perez, Tabosa y Silva 2018). Los libros de texto aún no presentan la ciencia como una actividad humana donde todos los niños y niñas crean que el día de mañana puedan llegar a trabajar como científicos o participar en la toma de decisiones de asuntos científicos que afectan al medio ambiente y a las generaciones fututas. Todavía hay deficiencias en el planteamiento realizado por esta herramienta educacional y un distanciamiento del marco competencial propuesto en el currículo oficial (Rodríguez-Moreno, J.; Pro-Bueno A. y Molina-Jaén 2018).

Los libros de texto actuales, además de incrementar las actividades que permitan a los estudiantes desarrollar las capacidades específicas de la práctica científica, deben plantear también otro tipo de actividades que impulsen actitudes científicas, valores relacionados con la ciencia y hábitos reflexivos sobre su importancia desde una perspectiva personal y social. La competencia científica integra las dimensiones conceptual, metodológica, actitudinal e integrada; contempla el desarrollo paulatino de capacidades, destrezas, habilidades, valores, actitudes y la comprensión de ideas y teorías científicas; para que los estudiantes puedan desarrollarla se requiere trabajar todas ellas de forma integrada y progresiva.

\section{Referencias}

Álvarez-Méndez, J. M. (2001). Entender la didáctica, entender el currículum. Madrid: Miño y Dávila.

ANELE (Asociación Nacional de Editores de Libros y Material de Enseñanza) (2013). Informe sobre la edición de libros de texto en España. Recuperado de http://www.anele.org/pdf/Sector\%20de\%20Libros\%20de\%20Texto\%202013.pdf

Anghel, B.; Cabrales, A. y Carro, J.M. (2013). Evaluación de un programa bilingüe en España: El impacto más allá del aprendizaje del idioma extranjero. Documento de trabajo. FEDEA. Universidad Carlos III. Madrid.

BOE (2014). R.D. 126/2014, de 28 de febrero, por el que se establece el currículo básico de la Educación Primaria

Cañal, P. (2012). Saber ciencias no equivale a tener competencia profesional para enseñar ciencias. En Once Ideas Clave. El desarrollo de la competencia científica, pp. 217-239 Barcelona: Graó.

Cristóbal-Aragón, E.; Greca, I.M. (2018) La enseñanza de las ciencias en un contexto bilingüe: propuesta para la enseñanza de contenidos de astronomía para primer ciclo de primaria. Revista de Enseñanz̧a de la Física 30 (2), 31-47.

Fraiha, S.; Paschoal, W.; Perez, S.; Tabosa, C. y Silva, C.R. (2018) Atividades indagativas e o desenvolvimento de habilidades e competências: um relato de experiencia no curso de Física da Universidade Federal do Pará. Rev. Brasileira de Ensino de Física 40(4) e4403.

Franco-Mariscal, A. J. (2015) Competencias científicas en la enseñanza y el aprendizaje por investigación. Un estudo de caso sobre corrosión de metales en secundaria. Enseñanz̧a de las Ciencias 33 (2), 231-252. 
García-Rodeja, I. (1997) ¿Qué propuestas de actividades hacen los libros de primaria? Alambique 11(1), 35-43.

Gómez, R. (2019). Estudio sobre el papel y valor de los contenidos educativos en las aulas. Grupo Análisis e Investigación. http://www.analisiseinvestigacion.com/

Greca I. M. y Meneses J.A. (2018). Proyectos STEAM en la educación primaria: fundamentos y aplicaciones prácticas. Madrid: DEXTRA.

Greca, I.M.; Díez-Ojeda, M.D. y Meneses-Villagrá, J.A. (2017) La formación en ciencias de los estudiantes del grado en maestro de Educación Primaria Rev. Electr. Enseñanza de Ciencias 16, 231.

Harlen, W (1998). Enseñanza y aprendizaje de las ciencias. Madrid: Morata

IAP. (InterAcademy Panel on International Issues) (2010). International Conference: Taking Inquiry-Based Science Education into the Sec- ondary School. Report available at: www.interacademies.net/File. aspx?id=15174

Laya-Iglesias, P. y Martínez-Losada, C. (2019) La competencia científica en los libros de texto de educación primaria. Ápice. Revista de Educación Científica 3 (1), 71.

López Valentín, D.M. y Guerra Ramos, M.T. (2013) Análisis de las actividades de aprendizaje incluidas en libros de texto de ciencias naturales para educación primaria utilizados en México. Enseñanza de las Ciencias, 31 (2), 173-179.

Martin Gámez, C., Prieto Ruz, T. y Jiménez López, M.A. (2013). El problema de la producción y el consumo de energía: ¿Cómo es tratado en los libros de texto de educación secundaria? . Enseñanza de las Ciencias 31 (2), 153-171

Martínez Losada, C. y García Barros, S. (2003a) Las actividades de primaria y ESO incluidas en libros escolares; ¿Qué objetivo persiguen? ¿Qué procedimientos enseñan? Enseñanz̧a de las Ciencias 21 (2), 243-264

Martínez Losada, C. y García Barros, S. (2003b) Análisis del trabajo práctico en textos escolares de primaria y secundaria. Enseñanza de las Ciencias, $n^{\circ}$ extra, 5-16.

Meneses Villagrá, J.A. y Caballero Sahelices, C. (2017) la metodología indagatoria en educación primaria. Una mirada desde la perspectiva del aprendizaje significativo. X Congreso Internacional sobre investigación en didáctica de las ciencias, Sevilla.

Moreno Martínez, L. y Calvo Pascual, M. A. (2019). ¿Cómo presentan la historia de la química los libros de texto de Educación Secundaria? Un análisis desde la didáctica y los estudios históricos de la ciencia. Rev. Eureka sobre Ens. y Divulg. Ciencias 16 (1), 1101

200National Research Council (1996). The National Science Education Standards, Washington D.C., The National Academies Press.

National Research Council (2000). Inquiry and the national science education standards: A guide for teaching and learning. Washington, D. C., The National Academies Press.

Occelli M. y Valeiras N. (2013) Los libros de texto de ciencias como objeto de investigación: una revisión bibliográfica. Enseñanza de las Ciencias 31 (2), 133-152.

Ortiz-Revilla, J.; Greca, I. M. y Adúriz-Bravo, A. (2018) El enfoque de enseñanza STEAM a través de la metodología de indagación. In Proyectos STEAM en la educación primaria: fundamentos y aplicaciones prácticas. Greca I. M. y Meneses J. A. (coord.). Madrid: Dextra. 
Park, D. y Lavonen, J. (2013) An analysis of standards-based high school physics textbooks of Finland and the United States. In M. S. Khine (Ed.) Critical Analysis of Science Textbooks: Evaluating Instructional Effectiveness. Dordrecht, The Netherlands: Springer.

Pedrinaci, E. (2012) El ejercicio de una ciudadanía responsable exige disponer de cierta competencia científica. En Once ideas clave. El desarrollo de la competencia científica, pp. 15-22. Barcelona: Graó.

Fraiha, S.; Paschoal, W.; Perez, S.; Tabosa, C. y Silva, C.R. (2018) Atividades indagativas e o desenvolvimento de habilidades e competências: um relato de experiencia no curso de Física da Universidade Federal do Pará. Rev. Brasileira de Ensino de Física 40(4) e4403.

PISA (2006). Marco de la evaluación. Conocimientos y habilidades en Ciencias, Matemáticas y Lectura. Madrid: Santillana Educación S.L

Rodríguez Moreno, J.; Pro Bueno A. y Molina Jaén, M. D. (2018) Opinión de los docentes sobre el tratamiento de las competencias en los libros de texto de Ciencias de la Naturaleza en Educación Primaria. Rev. Eureka sobre Ens. y Divulg. Ciencias 15 (3), 3102.

SEP (2009a). Plan de Estudios. Educación Básica. Primaria, México: SEP (2.a ed.).

Solas Ruiz, L. (2018) Introducción al concepto de fuerza a través de la construcción de una catapulta. In Proyectos STEAM en la educación primaria: fundamentos y aplicaciones prácticas. Greca I. M. y Meneses J. A. (coord..). Madrid: Dextra.

UNESCO (2010) Guidebook on Textbook Research and Textbook revision. United Nations Educational, Scientific and Cultural Organization.

Zômpero, A. F. y Laburú, C.E. (2011) Atividades investigativas no ensino de ciências: aspectos históricos e diferentes abordagens. Ensaio 13 (3), 67-80. 